\title{
Pre-pregnancy obesity and maternal nutritional biomarker status during pregnancy: a factor analysis
}

\author{
Laura E Tomedi ${ }^{1}$, Chung-Chou H Chang ${ }^{2}$, PK Newby ${ }^{3,4,5}$, Rhobert W Evans' ${ }^{1}$, \\ James $\mathrm{F}$ Luther ${ }^{1}$, Katherine LWisner ${ }^{1,6,7}$ and Lisa M Bodnar ${ }^{1,6,7, *}$ \\ ${ }^{1}$ Department of Epidemiology, University of Pittsburgh Graduate School of Public Health, 130 DeSoto Street, \\ Pittsburgh, PA 15261, USA: ${ }^{2}$ Department of Medicine, University of Pittsburgh School of Medicine, Pittsburgh, \\ PA, USA: ${ }^{3}$ Department of Pediatrics and Program in Medical Nutrition Sciences, Boston University School of \\ Medicine, Boston, MA, USA: ${ }^{4}$ Department of Epidemiology, Boston University School of Public Health, \\ Boston, MA, USA: ${ }^{5}$ Program in Gastronomy, Culinary Arts, and Wine Studies, Boston University Metropolitan \\ College, Boston, MA, USA: 'Western Psychiatric Institute and Clinic, University of Pittsburgh School of Medicine, \\ Pittsburgh, PA, USA: ${ }^{7}$ Department of Obstetrics, Gynecology, and Reproductive Sciences, University of Pittsburgh \\ School of Medicine, Pittsburgh, PA, USA
}

Submitted 3 August 2012: Final revision received 4 February 2013: Accepted 5 February 2013: First published online 25 March 2013

\begin{abstract}
Objective: Pre-pregnancy obesity has been associated with adverse birth outcomes. Poor essential fatty acid (EFA) and micronutrient status during pregnancy may contribute to these associations. We assessed the associations between pre-pregnancy BMI and nutritional patterns of maternal micronutrient and EFA status during mid-pregnancy.

Design: A cross-sectional analysis from a prospective cohort study. Women provided non-fasting blood samples at $\leq 20$ weeks' gestation that were assayed for red cell EFA; plasma folate, homocysteine and ascorbic acid; and serum retinol, 25-hydroxyvitamin $\mathrm{D}, \alpha$-tocopherol, soluble transferrin receptors and carotenoids. These nutritional biomarkers were employed in a factor analysis and three patterns were derived: EFA, Micronutrients and Carotenoids.

Setting: The Antidepressant Use During Pregnancy Study, Pittsburgh, PA, USA. Subjects: Pregnant women ( $n$ 129).

Results: After adjustment for parity, race/ethnicity and age, obese pregnant women were $3 \cdot 0(95 \%$ CI $1 \cdot 1,7 \cdot 7)$ times more likely to be in the lowest tertile of the EFA pattern and $4 \cdot 5(95 \%$ CI $1 \cdot 7,12 \cdot 3)$ times more likely to be in the lowest tertile of the Carotenoid pattern compared with their lean counterparts. We found no association between pre-pregnancy obesity and the Micronutrient pattern after confounder adjustment.

Conclusions: Our results suggest that obese pregnant women have diminished EFA and carotenoid concentrations.
\end{abstract}

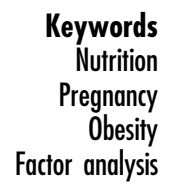

In the USA, nearly a quarter of all pregnancies are complicated by pre-pregnancy obesity and the prevalence is increasing ${ }^{(1)}$. Pregnant women who are obese are at higher risk of pre-eclampsia ${ }^{(2,3)}$, gestational diabetes mellitus ${ }^{(4)}$, birth trauma, large-for-gestational-age birth ${ }^{(2)}$ and stillbirth ${ }^{(2,5)}$ compared with their lean counterparts. Nevertheless, the mechanism by which obesity contributes to poor outcomes remains uncertain.

Nutritional status during pregnancy may partially mediate the relationship between pre-pregnancy obesity and adverse pregnancy and birth outcomes. In non-pregnant populations, obesity has been associated with insufficiencies of micronutrients, including vitamin $\mathrm{E}$, vitamin $\mathrm{C}$, vitamin $\mathrm{D}$, folate, vitamin $\mathrm{A}$ and carotenoids ${ }^{(6,7)}$. Obese individuals may also have lower levels of essential fatty acids $(\mathrm{EFA})^{(8,9)}$ than lean patients. But the relationship between obesity and nutritional biomarkers has not been thoroughly researched in pregnancy. Micronutrients and EFA play critical roles in the healthy physical and neurological development of the fetus and prevent conditions such as anaemia and neural tube defects ${ }^{(10,11)}$. Therefore, it is critical to explore these associations in pregnancy.

The objective of our study was to evaluate the association between pre-pregnancy BMI and patterns of nutritional biomarkers at $\leq 20$ weeks' gestation.

\section{Methods and procedures}

We conducted a secondary data analysis from the Antidepressant Use During Pregnancy (ADUP) Study, a 
prospective cohort study of pregnancy in Pittsburgh, PA, USA. The details of this study have been described previously ${ }^{(12,13)}$. Pregnant women were recruited at $\leq 20$ weeks' gestation. Eligible women had singleton gestations and women were excluded if they had psychosis, bipolar disorder, active substance use disorder, gestational exposure to benzodiazepines or prescription drugs in the category of $\mathrm{D}$ or $\mathrm{X}$ (other than selective serotonin reuptake inhibitors) defined by the US Food and Drug Administration, or pre-existing chronic diseases. The study was conducted according to the guidelines laid down in the Declaration of Helsinki and all procedures involving patients were approved by the University of Pittsburgh Institutional Review Board. Written informed consent was obtained from all patients.

The ADUP Study recruited women from 2000 to 2007. In 2004, the study protocol was modified to include nutrition measures, including biomarker assessment in maternal blood. Of the 197 eligible women interviewed from 2004 to 2007, 130 (66\%) provided a non-fasting blood sample at $\leq 20$ weeks that was processed for a full panel of nutritional biomarkers. We excluded one woman with missing data for pre-pregnancy weight. Our final analytic sample was 129 women. Women included in the analysis were less likely to be nulliparous than women who were excluded for missing weight measurements or blood samples $(33 \% v .51 \%, P=0 \cdot 01)$. Other maternal characteristics such as pre-pregnancy BMI, age, education, race, marital status, smoking status, employment status and diagnosed major depressive disorder did not differ significantly $(P>0 \cdot 05)$ between the two groups.

Our exposure of interest was general maternal adiposity before conception, measured using pre-pregnancy BMI (weight $(\mathrm{kg}) /$ height $(\mathrm{m})^{2}$ ). Pre-pregnancy BMI was based on pre-pregnancy weight, self-reported at enrolment, and measured height. Because only four women in the sample were underweight $\left(\mathrm{BMI}<18.5 \mathrm{~kg} / \mathrm{m}^{2}\right)$, we categorized women into three groups: lean (BMI $<25 \cdot 0 \mathrm{~kg} / \mathrm{m}^{2}$ ), overweight $\left(\mathrm{BMI}=25 \cdot 0-29 \cdot 9 \mathrm{~kg} / \mathrm{m}^{2}\right)$ and obese $(\mathrm{BMI} \geq 30 \cdot 0$ $\left.\mathrm{kg} / \mathrm{m}^{2}\right)^{(14)}$. Our outcome of interest was maternal nutritional status, as measured by patterns of nutritional biomarkers. At enrolment, women provided a non-fasting blood sample. Blood samples were assayed for red cell EFA, plasma folate, plasma homocysteine, plasma ascorbic acid, serum retinol, serum 25-hydroxyvitamin D, serum $\alpha$-tocopherol, serum ferritin, serum soluble transferrin receptors and serum carotenoids using methods described previously $^{(13,15)}$.

Women identified their race/ethnicity as non-Hispanic white, non-Hispanic African American and other. Because only four women self-identified as 'other', we combined the African American and the 'other' groups into a nonwhite category for analysis. Women were categorized as nulliparous or as having a previous live birth. A diagnosis of major depressive disorder was made using the Structured Clinical Interview for DSM-IV ${ }^{(16)}$. Educational status was defined as having less than a high school education, some college, college degree and post-graduate education. Women were classified as unemployed or employed (which included women who worked full time, part time or occasionally). For marital status, 'married' included married or living as married and 'unmarried' included single, separated, widowed and divorced. Women were classified as current smokers if they smoked at all during pregnancy or non-smokers if they did not.

We used Pearson $\chi^{2}$ tests and Student $t$ tests to determine differences in maternal characteristics by pre-pregnancy BMI categories. To describe skewed biomarkers, we calculated geometric means and log-transformed biomarkers before statistical testing. We conducted a factor analysis on the fifteen untransformed maternal dietary biomarkers as described previously ${ }^{(13)}$. Three patterns were generated and assigned names based on the biomarkers that loaded heavily on the pattern. Each represents biologically meaningful correlations between biomarkers: pattern 1 'Essential Fatty Acids (EFA)', pattern 2 'Micronutrients' and pattern 3 'Carotenoids'. Pattern scores were categorized based on tertiles of the distribution.

Logistic regression was used to assess the independent associations between pre-pregnancy BMI and the likelihood of being in the lowest tertile of each nutritional pattern. Potential confounders were maternal age, race/ ethnicity, parity, education, marital status, smoking status, employment and depression (diagnosed with the Structured Clinical Interview for DSM-IV $\left.{ }^{(16)}\right)$. Only parity, race/ethnicity and age met our a priori definition of confounding ( $\geq 10 \%$ change in the odds ratio after excluding the covariate from the full model). Analyses were conducted using statistical software package Stata version $11 \cdot 0$.

\section{Results}

The women in the cohort were primarily well-educated, non-Hispanic white, married, non-smokers and employed (Table 1). The mean pre-pregnancy BMI was $26 \cdot 6$ (SD 6.0) $\mathrm{kg} / \mathrm{m}^{2}$. About half (49.6\%) of the women were lean, $22.5 \%$ were overweight and $27 \cdot 9 \%$ were obese. Obese women were less likely to have a college degree $(P<0 \cdot 01)$ and more likely to be nulliparous $(P<0 \cdot 01)$ and depressed $(P=0 \cdot 03)$ than lean women. Maternal age, race/ethnicity, marital status, smoking and employment did not differ significantly by pre-pregnancy BMI category.

In unadjusted analysis, women who were overweight before pregnancy had lower mean concentrations of plasma folate $(P=0 \cdot 04)$, plasma ascorbic acid $(P=0 \cdot 01)$, serum $\beta$-carotene $(P=0 \cdot 01)$ and serum $\beta$-cryptoxanthin $(P=0 \cdot 02)$ than lean women (Table 2$)$. Compared with lean women, obese pregnant women had lower mean concentrations of red cell DHA $(P<0 \cdot 01)$, red cell arachidonic acid $(P=0 \cdot 01)$, plasma ascorbic acid $(P=0 \cdot 03)$, 
Table 1 Maternal characteristics of the study population, stratified by pre-pregnancy BMl; Antidepressant Use During Pregnancy Study, Pittsburgh, PA, USA

\begin{tabular}{|c|c|c|c|c|c|c|c|c|c|}
\hline \multirow[b]{2}{*}{ Characteristic } & \multicolumn{2}{|c|}{ Total $(n$ 129) } & \multicolumn{2}{|c|}{$\mathrm{BMI}<25 \cdot 0 \mathrm{~kg} / \mathrm{m}^{2}(n 64)$} & \multicolumn{2}{|c|}{$\mathrm{BMI}=25 \cdot 0-29 \cdot 9 \mathrm{~kg} / \mathrm{m}^{2}(n 29)$} & \multicolumn{2}{|c|}{$\mathrm{BMI} \geq 30 \cdot 0 \mathrm{~kg} / \mathrm{m}^{2}(n 36)$} & \multirow[b]{2}{*}{$P$ value* } \\
\hline & $n$ & $\%$ & $n$ & $\%$ & $n$ & $\%$ & $n$ & $\%$ & \\
\hline Age (years) & \multirow{3}{*}{\multicolumn{2}{|c|}{$\begin{array}{r}30 \cdot 3 \\
5 \cdot 6\end{array}$}} & & & & & & & $0 \cdot 14$ \\
\hline Mean & & & \multirow{2}{*}{\multicolumn{2}{|c|}{$\begin{array}{r}30 \cdot 6 \\
5 \cdot 8\end{array}$}} & \multirow{2}{*}{\multicolumn{2}{|c|}{$31 \cdot 6$}} & \multirow{2}{*}{\multicolumn{2}{|c|}{$28 \cdot 7$}} & \\
\hline SD & & & & & & & & & \\
\hline \multicolumn{10}{|l|}{ Race/ethnicity } \\
\hline White & 103 & $79 \cdot 8$ & 56 & $87 \cdot 5$ & 23 & $79 \cdot 3$ & 24 & $66 \cdot 7$ & \\
\hline African American & 22 & $17 \cdot 1$ & 6 & $9 \cdot 4$ & 6 & $20 \cdot 7$ & 10 & $27 \cdot 8$ & \\
\hline Other & 4 & $3 \cdot 1$ & 2 & $3 \cdot 1$ & 0 & $0 \cdot 0$ & 2 & $5 \cdot 6$ & $0 \cdot 10$ \\
\hline \multicolumn{10}{|l|}{ Parity } \\
\hline 0 & 43 & $33 \cdot 3$ & 33 & $51 \cdot 6$ & 23 & $79 \cdot 3$ & 30 & $83 \cdot 3$ & \\
\hline $1-6$ & 86 & $66 \cdot 7$ & 31 & $48 \cdot 4$ & 6 & $20 \cdot 7$ & 6 & $16 \cdot 7$ & $<0.01$ \\
\hline \multicolumn{10}{|l|}{ Educational status } \\
\hline$\leq$ High school & 23 & $17 \cdot 8$ & 5 & $7 \cdot 8$ & 4 & $13 \cdot 8$ & 14 & $38 \cdot 9$ & \\
\hline Some college & 25 & $19 \cdot 4$ & 12 & $18 \cdot 8$ & 4 & $13 \cdot 8$ & 9 & $25 \cdot 0$ & \\
\hline College degree & 45 & 34.9 & 25 & $39 \cdot 1$ & 9 & $31 \cdot 0$ & 11 & $30 \cdot 6$ & \\
\hline Post-graduate education & 36 & $27 \cdot 9$ & 22 & $34 \cdot 4$ & 12 & $41 \cdot 4$ & 2 & $5 \cdot 6$ & $<0.01$ \\
\hline \multicolumn{10}{|l|}{ Marital status } \\
\hline Married & 95 & $73 \cdot 6$ & 48 & $75 \cdot 0$ & 25 & $86 \cdot 2$ & 22 & $61 \cdot 1$ & \\
\hline Unmarried & 34 & $26 \cdot 4$ & 16 & $25 \cdot 0$ & 4 & $13 \cdot 8$ & 14 & $38 \cdot 9$ & 0.07 \\
\hline \multicolumn{10}{|l|}{ Smoking status } \\
\hline Non-smoker & 110 & $85 \cdot 3$ & 56 & $87 \cdot 5$ & 27 & $93 \cdot 1$ & 27 & $75 \cdot 0$ & \\
\hline Smoker & 19 & $14 \cdot 7$ & 8 & $12 \cdot 5$ & 2 & $6 \cdot 9$ & 9 & $25 \cdot 0$ & $0 \cdot 10$ \\
\hline \multicolumn{10}{|l|}{ Employment status } \\
\hline Employed & 73 & $56 \cdot 6$ & 37 & $57 \cdot 8$ & 17 & $58 \cdot 6$ & 19 & $52 \cdot 8$ & \\
\hline Unemployed & 56 & $43 \cdot 4$ & 27 & $42 \cdot 2$ & 12 & $41 \cdot 4$ & 17 & $47 \cdot 2$ & 0.86 \\
\hline \multicolumn{10}{|l|}{ Major depressiont } \\
\hline Not depressed & 96 & $74 \cdot 4$ & 53 & $82 \cdot 8$ & 22 & 75.9 & 21 & $58 \cdot 3$ & \\
\hline Depressed & 33 & $25 \cdot 6$ & 11 & $17 \cdot 2$ & 7 & $24 \cdot 1$ & 15 & $41 \cdot 7$ & 0.03 \\
\hline
\end{tabular}

*Based on Student's $t$ test for maternal age and $\chi^{2}$ test for the other covariates.

tAs measured by the Structured Clinical Interview for DSM-IV ${ }^{(16)}$.

Table 2 Mean* maternal nutritional biomarkers at 20 weeks' gestation, stratified by pre-pregnancy BMl; Antidepressant Use During Pregnancy Study, Pittsburgh, PA, USA

\begin{tabular}{|c|c|c|c|c|c|c|c|c|}
\hline \multirow[b]{2}{*}{ Biomarker } & \multicolumn{2}{|c|}{$\mathrm{BMI}<25 \cdot 0 \mathrm{~kg} / \mathrm{m}^{2}(n 64)$} & \multicolumn{2}{|c|}{$\mathrm{BMI}=25 \cdot 0-29 \cdot 9 \mathrm{~kg} / \mathrm{m}^{2}(n 29)$} & \multirow[b]{2}{*}{$P$ valuet } & \multicolumn{2}{|c|}{$\mathrm{BMI} \geq 30 \cdot 0 \mathrm{~kg} / \mathrm{m}^{2}(n 36)$} & \multirow[b]{2}{*}{$P$ value } \\
\hline & Mean & $95 \% \mathrm{Cl}$ & Mean & $95 \% \mathrm{Cl}$ & & Mean & $95 \% \mathrm{Cl}$ & \\
\hline Red cell DHA (\%) & $3 \cdot 4$ & $2 \cdot 7,4 \cdot 4$ & $3 \cdot 1$ & $2 \cdot 2,4 \cdot 2$ & 0.58 & $1 \cdot 9$ & $1 \cdot 3,2 \cdot 7$ & $<0.01$ \\
\hline Red cell AA (\%) & $13 \cdot 0$ & $11 \cdot 4,14 \cdot 8$ & $12 \cdot 9$ & $10 \cdot 5,15 \cdot 8$ & 0.95 & $9 \cdot 3$ & $7 \cdot 3,11 \cdot 9$ & 0.01 \\
\hline Red cell EPA (\%) & 0.33 & $0.28,0.40$ & 0.28 & $0.22,0.35$ & $0 \cdot 30$ & $0 \cdot 25$ & $0.18,0.33$ & 0.06 \\
\hline Plasma folate $(\mathrm{ng} / \mathrm{ml})$ & $15 \cdot 3$ & $13 \cdot 6,17 \cdot 2$ & $12 \cdot 5$ & $10 \cdot 7,14 \cdot 6$ & 0.04 & $13 \cdot 0$ & $11 \cdot 3,15 \cdot 1$ & 0.08 \\
\hline Plasma ascorbic acid $(\mu \mathrm{g} / \mathrm{ml})$ & $12 \cdot 9$ & $12 \cdot 1,13 \cdot 7$ & $10 \cdot 4$ & $8 \cdot 7,12 \cdot 4$ & 0.01 & $11 \cdot 0$ & $9 \cdot 7,12 \cdot 4$ & 0.03 \\
\hline Serum retinol $(\mu \mathrm{g} / \mathrm{ml})$ & $0 \cdot 50$ & $0.48,0.53$ & $0 \cdot 46$ & $0.43,0.50$ & $0 \cdot 11$ & 0.47 & $0.43,0.51$ & $0 \cdot 17$ \\
\hline Serum $25(\mathrm{OH}) \mathrm{D}(\mathrm{nmol} / \mathrm{l})$ & $88 \cdot 5$ & $81 \cdot 3,96 \cdot 3$ & $77 \cdot 8$ & $63 \cdot 1,96 \cdot 0$ & $0 \cdot 20$ & 69.9 & $59 \cdot 0,82 \cdot 9$ & 0.01 \\
\hline Serum $\alpha$-tocopherol $(\mu \mathrm{g} / \mathrm{ml})$ & 0.07 & $0.06,0.07$ & 0.07 & $0.06,0.08$ & $0 \cdot 72$ & 0.07 & $0.06,0.07$ & 0.47 \\
\hline Serum $\beta$-carotene $(\mu \mathrm{g} / \mathrm{ml})$ & $0 \cdot 14$ & $0 \cdot 10,0 \cdot 19$ & 0.06 & $0.04,0 \cdot 11$ & 0.01 & 0.04 & $0.03,0.06$ & $<0.01$ \\
\hline Serum homocysteine $(\mu \mathrm{mol} / \mathrm{l})$ & $2 \cdot 3$ & $2 \cdot 1,2 \cdot 6$ & $2 \cdot 3$ & $2 \cdot 1,2 \cdot 6$ & 0.94 & $2 \cdot 3$ & $1 \cdot 9,2 \cdot 7$ & 0.75 \\
\hline Serum sTfR (nmol/l) & $14 \cdot 5$ & $13 \cdot 7,15 \cdot 4$ & $15 \cdot 9$ & $14 \cdot 5,17 \cdot 4$ & 0.09 & $16 \cdot 3$ & $14 \cdot 9,17 \cdot 7$ & 0.03 \\
\hline Serum lutein + zeaxanthin $(\mu \mathrm{g} / \mathrm{ml})$ & $0 \cdot 14$ & $0 \cdot 12,0.17$ & 0.12 & $0 \cdot 10,0 \cdot 14$ & $0 \cdot 17$ & 0.08 & $0.06,0.10$ & $<0.01$ \\
\hline Serum $\beta$-cryptoxanthin $(\mu \mathrm{g} / \mathrm{ml})$ & $0 \cdot 10$ & $0 \cdot 08,0 \cdot 13$ & 0.06 & $0.04,0.09$ & 0.02 & 0.05 & $0.04,0.07$ & $<0.01$ \\
\hline Serum lycopene $(\mu \mathrm{g} / \mathrm{ml})$ & $0 \cdot 30$ & $0.26,0.35$ & 0.32 & $0.27,0.37$ & $0 \cdot 76$ & 0.28 & $0.23,0.34$ & 0.41 \\
\hline
\end{tabular}

AA, arachidonic acid; $25(\mathrm{OH}) \mathrm{D}, 25$-hydroxyvitamin $\mathrm{D}$; sTfR, soluble transferrin receptors.

${ }^{*}$ Geometric means, all values are presented as mean and $95 \%$ confidence interval.

tStudent's $t$ test, biomarkers log-transformed before tests were performed, $\mathrm{BMI}<25 \cdot 0 \mathrm{~kg} / \mathrm{m}^{2}$ is the reference group.

serum 25-hydroxyvitamin $\mathrm{D}(P=0 \cdot 01)$, serum $\beta$-carotene $(P<0 \cdot 01)$, serum lutein + zeaxanthin $(P<0 \cdot 01)$ and serum $\beta$-cryptoxanthin $(P<0 \cdot 01)$ and higher mean serum soluble transferrin receptors concentration $(P=0 \cdot 03)$.

In unadjusted analysis, obese women had significantly greater odds of being in the lowest tertile of all three nutritional patterns. After adjustment for confounders, obese pregnant women were three and five times more likely to be in the lowest tertile of the EFA and Carotenoid pattern, respectively, than lean women (Table 3). After adjustment, there was no association between obesity and Micronutrient pattern score. There was no relationship between pre-pregnancy overweight and any pattern before or after adjustment. 
Table 3 Association between pre-pregnancy BMI and the lowest tertile* of each nutritional pattern; Antidepressant Use During Pregnancy Study, Pittsburgh, PA, USA

\begin{tabular}{|c|c|c|c|c|c|c|}
\hline & \multicolumn{6}{|c|}{ Nutrient pattern ( $n$ 129) } \\
\hline & \multicolumn{2}{|c|}{ Essential Fatty Acids } & \multicolumn{2}{|c|}{ Micronutrient } & \multicolumn{2}{|c|}{ Carotenoid } \\
\hline & OR & $95 \% \mathrm{Cl}$ & OR & $95 \% \mathrm{Cl}$ & OR & $95 \% \mathrm{Cl}$ \\
\hline \multicolumn{7}{|l|}{ Unadjusted model } \\
\hline $\mathrm{BMI}<25 \cdot 0 \mathrm{~kg} / \mathrm{m}^{2}$ & \multicolumn{2}{|c|}{ Ref. } & \multicolumn{2}{|c|}{ Ref. } & \multicolumn{2}{|c|}{ Ref. } \\
\hline $\mathrm{BMI}=25 \cdot 0-29.9 \mathrm{~kg} / \mathrm{m}^{2}$ & $1 \cdot 6$ & $0 \cdot 6,4 \cdot 1$ & $2 \cdot 5$ & $1 \cdot 0,6 \cdot 5$ & $1 \cdot 4$ & $0.5,3.7$ \\
\hline $\mathrm{BMI} \geq 30 \cdot 0 \mathrm{~kg} / \mathrm{m}^{2}$ & $2 \cdot 7$ & $1 \cdot 1,6 \cdot 4$ & $3 \cdot 6$ & $1 \cdot 5,8 \cdot 6$ & $5 \cdot 0$ & $2 \cdot 1,12 \cdot 2$ \\
\hline \multicolumn{7}{|l|}{ Adjusted modelt } \\
\hline $\mathrm{BMI}<25 \cdot 0 \mathrm{~kg} / \mathrm{m}^{2}$ & \multicolumn{2}{|c|}{ Ref. } & \multicolumn{2}{|c|}{ Ref. } & \multicolumn{2}{|c|}{ Ref. } \\
\hline $\mathrm{BMI}=25 \cdot 0-29 \cdot 9 \mathrm{~kg} / \mathrm{m}^{2}$ & 1.5 & $0 \cdot 6,4 \cdot 1$ & $1 \cdot 8$ & $0 \cdot 6,5 \cdot 7$ & $1 \cdot 4$ & $0 \cdot 5,4 \cdot 3$ \\
\hline $\mathrm{BMI} \geq 30 \cdot 0 \mathrm{~kg} / \mathrm{m}^{2}$ & $3 \cdot 0$ & $1 \cdot 1,7 \cdot 7$ & 1.5 & $0 \cdot 5,4 \cdot 5$ & $4 \cdot 5$ & $1 \cdot 7,12 \cdot 3$ \\
\hline
\end{tabular}

Ref, referent category.

*Lowest tertile compared to the combined middle and highest tertiles of nutrient components.

tAdjusted for parity, race/ethnicity and age. Further adjustment for other covariates had no meaningful impact on the findings.

\section{Discussion}

Using factor analysis of an array of nutritional biomarkers, we found that a larger percentage of obese women were in the lowest tertile of the EFA, Micronutrient and Carotenoid patterns than lean women at $\leq 20$ weeks' gestation. After adjustment for maternal characteristics, pre-pregnancy obesity remained associated with poorer EFA and Carotenoid patterns.

To our knowledge, the present study is the first one to examine pre-pregnancy BMI relative to a wide range of maternal biomarkers. Our EFA and carotenoid conclusions are in agreement with previous studies of individual biomarkers in a variety of non-pregnant populations ${ }^{(7,17-20)}$. For example, in a cross-sectional analysis of 4512 nonpregnant women, both obese and overweight women were significantly more likely to be in the lowest 20th percentile of a sum of carotenoid concentrations than lean women ${ }^{(6)}$. Although we observed that obesity was associated with lower Micronutrient pattern scores in unadjusted analysis, adjustment for parity, race/ethnicity and age eliminated this effect. In contrast, other researchers have reported poorer micronutrient concentrations among obese women in nonpregnant populations ${ }^{(6,21)}$. One study of non-pregnant women found that obese women were more likely to have low levels of vitamin E, vitamin C, vitamin D and serum folate than non-obese women ${ }^{(6)}$. Studies in pregnancy report that maternal obesity increases the likelihood of poor maternal vitamin $\mathrm{D}^{(22)}$ and folate ${ }^{(23)}$ status in pregnancy. The differences in our conclusions may be due in part to prenatal vitamin use. While prenatal vitamins always contain micronutrients, they do not uniformly contain EFA or many carotenoids. Given the high socio-economic status of our population, the use of prenatal vitamins and other dietary supplements may have been widespread, leading to a predominance of elevated micronutrient concentrations. If obese women in our cohort were taking prenatal supplements but consumed diets poor in fish, the major source of DHA and EPA, or fruits and vegetables, which contribute to carotenoids ${ }^{(24)}$, this may account for our results. Unfortunately, complete data on dietary intake and supplementation were not available at 20 weeks' gestation in the ADUP Study.

In addition to diet and supplementation differences between obese and lean pregnant women, pre-pregnancy obesity may alter the absorption and metabolism of carotenoids and EFA. It has been previously hypothesized that fat-soluble nutrients, such as carotenoids, may also be sequestered by adipose tissue ${ }^{(25)}$. However, among the individual nutritional biomarkers that had lower concentrations in obese women, we did not find a distinct pattern between the lipid-soluble and water-soluble markers.

Major strengths of our study include an analysis of a wide array of measured nutritional biomarkers. The use of factor analysis accounted for inherent correlations between nutrients and reduced the likelihood of type 1 error due to multiple comparisons. Our study was limited by self-reported weight to calculate BMI, which may have contributed to non-differential misclassification ${ }^{(26)}$. While we had a relatively small sample size of primarily white, well-educated, non-smoking women, we do not expect the biological associations between nutritional biomarkers and pre-pregnancy BMI to alter based on these factors. Our lack of longitudinal biomarker measurements limits our understanding of the temporality of these relationships. Large, prospective studies with repeated biomarker measures and detailed information on supplement use during pregnancy are needed to determine the extent of the association between maternal obesity and poor nutritional status.

Our results suggest that obese pregnant women have diminished EFA and carotenoid status during pregnancy. A better understanding of the effect of pre-pregnancy obesity on maternal micronutrient and EFA status may lead to specific nutritional interventions that can improve pregnancy and birth outcomes in this at-risk population. 


\section{Acknowledgements}

Sources offunding: Support for this project came from the National Institutes of Health (grants K01 MH074092, R01 MH060335 and R24 HL076858) and the Eunice Kennedy Shriver National Institute of Child Health \& Human Development (Reproductive, Perinatal and Pediatric Epidemiology Grant HD 055 162-03 NIH NRSA T32). Conflicts of interest: The authors have no conflicts of interest to declare. Ethics: The study was approved by the University of Pittsburgh Institutional Review Board. Authors' contributions: L.E.T. analysed the data and wrote the manuscript; C.-C.H.C. provided valuable biostatistical assistance; P.K.N. provided significant assistance with pattern analysis; R.W.E. provided technical assistance for the biomarker assays and critically reviewed the manuscript; J.F.L. performed the principal component analysis; K.L.W. designed the research and provided the data; L.M.B. provided significant analytical assistance and critically edited the manuscript; all authors read and approved the final manuscript. Acknowledgements: The authors thank Ms Brandi Duffy and Ms Rona de la Vega for expert chemical analysis.

\section{References}

1. Kim SY, Dietz PM, England L et al. (2007) Trends in pre-pregnancy obesity in nine states, 1993-2003. Obesity (Silver Spring) 15, 986-993.

2. Cedergren MI (2004) Maternal morbid obesity and the risk of adverse pregnancy outcome. Obstet Gynecol 103, 219-224.

3. Hauger MS, Gibbons L, Vik T et al. (2008) Prepregnancy weight status and the risk of adverse pregnancy outcome. Acta Obstet Gynecol Scand 87, 953-959.

4. Chu SY, Callaghan WM, Kim SY et al. (2007) Maternal obesity and risk of gestational diabetes mellitus. Diabetes Care 30, 2070-2076.

5. Cnattingius S, Bergstrom R, Lipworth L et al. (1998) Prepregnancy weight and the risk of adverse pregnancy outcomes. N Engl J Med 338, 147-152.

6. Kimmons JE, Blanck HM, Tohill BC et al. (2006) Associations between body mass index and the prevalence of low micronutrient levels among US adults. MedGenMed 8, 59.

7. Andersen LF, Jacobs DR Jr, Gross MD et al. (2006) Longitudinal associations between body mass index and serum carotenoids: the CARDIA study. Br J Nutr 95, 358-365.

8. Kishino T, Watanabe K, Urata $\mathrm{T}$ et al. (2008) Visceral fat thickness in overweight men correlates with alterations in serum fatty acid composition. Clin Chim Acta 398, 57-62.

9. Karlsson M, Marild S, Brandberg J et al. (2006) Serum phospholipid fatty acids, adipose tissue, and metabolic markers in obese adolescents. Obesity (Silver Spring) 14, 1931-1939.

10. Institute of Medicine (1990) Nutrition During Pregnancy: Part I, Weight Gain: Part II, Nutrient Supplements. Washington, DC: National Academy Press.

11. Uauy R, Hoffman DR, Peirano P et al. (2001) Essential fatty acids in visual and brain development. Lipids 36, 885-895.

12. Wisner KL, Sit DK, Hanusa BH et al. (2009) Major depression and antidepressant treatment: impact on pregnancy and neonatal outcomes. Am J Psychiatry 166, 557-566.

13. Bodnar LM, Wisner KL, Luther JF et al. (2012) An exploratory factor analysis of nutritional biomarkers associated with major depression in pregnancy. Public Health Nutr 15, 1078-1086.

14. National Institutes of Health (1998) Clinical Guidelines on the Identification, Evaluation, and Treatment of Overweight and Obesity in Adults - The Evidence Report. Obes Res 6, Suppl. 2, 51S-209S.

15. Tomedi LE, Bogen DL, Hanusa BH et al. (2012) A pilot study of the nutritional status of opiate-using pregnant women on methadone maintenance therapy. Subst Use Misuse 47, 286-295.

16. First MB, Gibbon M, Spitzer RL et al. (1997) Structured Clinical Interview for DSM-IV Axis II Personality Disorders (SCID-II). Washington, DC: American Psychiatric Press, Inc.

17. Galan P, Viteri FE, Bertrais S et al. (2005) Serum concentrations of $\beta$-carotene, vitamins $\mathrm{C}$ and $\mathrm{E}$, zinc and selenium are influenced by sex, age, diet, smoking status, alcohol consumption and corpulence in a general French adult population. Eur J Clin Nutr 59, 1181-1190.

18. Chai W, Conroy SM, Maskarinec G et al. (2010) Associations between obesity and serum lipid-soluble micronutrients among premenopausal women. Nutr Res 30, 227-232.

19. Sands SA, Reid KJ, Windsor SL et al. (2005) The impact of age, body mass index, and fish intake on the EPA and DHA content of human erythrocytes. Lipids 40, 343-347.

20. Micallef M, Munro I, Phang M et al. (2009) Plasma $n-3$ polyunsaturated fatty acids are negatively associated with obesity. Br J Nutr 102, 1370-1374

21. Aasheim ET, Hofso D, Hjelmesaeth J et al. (2008) Vitamin status in morbidly obese patients: a cross-sectional study. Am J Clin Nutr 87, 362-369.

22. Bodnar LM, Catov JM, Roberts JM et al. (2007) Prepregnancy obesity predicts poor vitamin $\mathrm{D}$ status in mothers and their neonates. J Nutr 137, 2437-2442.

23. Han YS, Ha EH, Park HS et al. (2011) Relationships between pregnancy outcomes, biochemical markers and pre-pregnancy body mass index. Int J Obes (Lond) 35, 570-577.

24. Laraia BA, Bodnar LM \& Siega-Riz AM (2007) Pregravid body mass index is negatively associated with diet quality during pregnancy. Public Health Nutr 10, 920-926.

25. Wallstrom P, Wirfalt E, Lahmann PH et al. (2001) Serum concentrations of $\beta$-carotene and $\alpha$-tocopherol are associated with diet, smoking, and general and central adiposity. Am J Clin Nutr 73, 777-785.

26. Bodnar LM, Siega-Riz AM, Simhan HN et al. (2010) The impact of exposure misclassification on associations between prepregnancy BMI and adverse pregnancy outcomes. Obesity (Silver Spring) 18, 2184-2190. 